\title{
Designing Joint Precoding and Beamforming in a Multiple Gateway Multibeam Satellite System
}

\author{
Vahid Joroughi*, Bhavani Shankar M.R.*, Sina Maleki*, Symeon Chatzinotas* Joel Grotz ${ }^{\dagger}$ and Björn Ottersten* \\ ${ }^{*}$ Interdisciplinary Centre for Security, Reliability and Trust (SnT), the University of Luxembourg, Luxembourg. \\ ${ }^{\dagger}$ SES S.A., Betzdolf, Luxembourg. \\ Email: \{vahid.joroughi, bhavani.shankar, sina.maleki, symeon.chatzinotas, bjorn.ottersten\}@uni.lu, joel.grotz@ses.com
}

\begin{abstract}
This paper aims to design joint on-ground precoding and on-board beamforming of a multiple gateway multibeam satellite system in a hybrid space-ground mode where full frequency reuse pattern is considered among the beams. In such an architecture, each gateway serves a cluster of adjacent beams such that the adjacent clusters are served through a set of gateways that are located at different geographical areas. However, such a system brings in two challenges to overcome. First, the inter-beam interference is the bottleneck of the whole system and applying interference mitigation techniques becomes necessary. Second, as the data demand increases, the ground and space segments should employ extensive bandwidth resources in the feeder link accordingly. This entails embedding an extra number of gateways aiming to support a fair balance between the increasing demand and the corresponding required feeder link resources. To solve these problems, this study investigates the impact of employing a joint multiple gateway architecture and onboard beamforming scheme. It is shown that by properly designing the on-board beamforming scheme, the number of gateways can be kept affordable even if the data demand increases. Moreover, Zero Forcing (ZF) precoding technique is considered to cope with the inter-beam interference where each gateway constructs a part of block $\mathrm{ZF}$ precoding matrix. The conceived designs are evaluated with a close-to-real beam pattern and the latest broadband communication standard for satellite communications.
\end{abstract}

Index Terms-Multibeam satellite systems, multiple gateway systems, on-board beamforming, precoding techniques.

\section{INTRODUCTION}

\section{A. Motivation}

Built on the Multiuser Multiple-Input Multiple-Output (MUMIMO) framework, the use of multiple spot beams in modern broadband satellites have been recently considered by employing fractional frequency reuse among beams. Such systems rely on employing a large number of spot beams instead of a single (global) beam in the coverage area to provide higher spectral efficiency [1]. However, one of the major challenges in multibeam architecture is how to deal with interference in the access network. Indeed, adjacent beams create high levels of interference due to the side lobes of the radiation pattern of beams on the Earth surface. A promising technique in this context is to use full frequency reuse pattern among the beams by resorting interference mitigation techniques as precoding in the forward link and multi-user detection in the return link [2].

Apart from the already mentioned interference limitation, another major challenge of multibeam systems is to deal with the large spectral demands on the Feeder Link (FL), i.e. the bidirectional link between satellite and the Gateway (GW), whose bandwidth requirements increase as it aggregates the traffic of all users. Keeping a full frequency reuse allocation, the required FL resources can be calculated as

$$
\mathrm{B}_{\text {feeder-link }}=N \mathrm{~B}_{\text {beam }},
$$

where $N$ is the number of on-board feed signals. The notations $\mathrm{B}_{\text {beam }}$ and $\mathrm{B}_{\text {feeder-link }}$ are the per-beam and the FL required bandwidths, respectively. Let us consider a total number of $K$ beams with $N>K$. From (1), it is evident that any beam available bandwidth enhancement forces the FL resources to be increased accordingly and, eventually the FL might become the communication bottleneck. Note that, in contrast to the single feed per beam architectures, i.e. $N=K$, applying Multiple Feeds per Beam (MFB) at the payload, i.e. $N>K$, can reduce the scan losses for a large continental coverage, and are specially suited for contour beams [1]. Recently, some techniques have been proposed in order to reduce the FL spectrum requirements. One solution is moving the FL from the $\mathrm{Ka}$ band to the $\mathrm{Q} / \mathrm{V}$ band so that a larger available bandwidths can be achieved [3]. Unfortunately, the $\mathrm{Q} / \mathrm{V}$ carrier frequencies suffer the impact of an extremely large fading and more advanced transmitting schemes at the GW are needed.

Toward optimizing the FL resources, a Beamforming Network (BFN) at the payload can be employed aiming at: i) synthesizing the amplitude and phase modulating the excitation of each on-borad feed in the MFB scheme, ii) reducing the FL bandwidth requirements [4] by

$$
\mathrm{B}_{\text {feeder-link-onboard }}=K \mathrm{~B}_{\text {beam }} \text {, }
$$

where $B_{\text {feeder-link-onboard }}$ denotes the FL resources that is required after employing the on-board BFN with $\mathrm{B}_{\text {feeder-link-onboard }}<\mathrm{B}_{\text {feeder-link }}$ and $N>K$. Despite the slight improvement, the FL resources still vary linearly with the number of beams.

Another promising option is the use of on-ground multiple GW architecture. This architecture exploits the multiplexing diversity by reusing all the available FL bandwidth across the different GWs [5]. In this context, the required FL bandwidth becomes

$$
\mathrm{B}_{\text {feeder-link-MG }}=\frac{N}{F} \mathrm{~B}_{\text {beam }},
$$

where $F$ is the number of GWs, and $\mathrm{B}_{\text {feeder-link-MG }}$ denotes the FL resources which is required at multiple GW architecture. Indeed, the multiple $\mathrm{GW}$ architecture reduces the required FL resources to $\mathrm{B}_{\text {feeder-link-MG }}<\mathrm{B}_{\text {feeder-link-onboard }}$ with $\frac{N}{F}<K$. Nevertheless, the deployment of several GWs increases the cost of the system. Besides, considering (3), by increasing the demand in the coverage area, a specific number of GWs shall be employed aiming to provide a fair balance between the increased demands and their required FL resources.

\section{B. Contributions}

This paper investigates the forward link of a novel multiple GW architecture where a BFN scheme is applied at the payload such that the FL requirements in (3) reduce to

$$
\mathrm{B}_{\text {feeder-link-onboard-MG }}=\frac{K}{F} \mathrm{~B}_{\text {beam }},
$$

with $\mathrm{B}_{\text {feeder-link-onboard-MG }}<\mathrm{B}_{\text {feeder-link-MG }}$ in (3) and $N>K$. This is referred as joint BFN and Multiple Gateway Processing (BMGP) multibeam network. In addition, since the channel components of 
the User Link (UL), i.e. the bidirectional link between satellite and GWs, are intuitively varied, this paper focuses on the presence of a fixed BFN in the proposed BMGP architecture which is sufficiently robust to the variations in the UL, aiming at preserving the payload complexity affordable.

Remark 1: Throughout this paper the variability of the UL channel components is due to the change of position of the users in consecutive time instants and atmospheric fading.

To manage the interference of the UL in our proposed BMGP architecture, it is considered that the GWs perform precoding on the transmitted signals by a Zero Forcing (ZF) precoding technique so that each GW computes a part of the full precoding matrix. For the sake of preserving low complexity, we develop the ZF precoding scheme while the perfect CSI of the UL is available at the GWs through the satellite. This assumption comes from the fact that a perfect CSI feedback (with no quantization errors) regime and also a noiseless CSI exchange mechanism among GWs is established in the infrastructure of BMGP architecture. Quantazing the perfect CSI at GWs is beyond the scope of this work.

It should be also noted that even with highly directive antennas the FL originating at different GWs are partially interfering. Nevertheless, in this work we assume that GWs are sufficiently separated on the Earth surface and space so that the inter-feeder link interference can be ignored.

The rest of paper is organized as follows. Section II presents the considered scenario. The precoding and BFN schemes are developed in Sections III. Section IV provides numerical results. Finally, we derive our conclusion in Section V.

Notation: In this paper, the following notation are adopted. Boldface uppercase letters denote matrices and boldface lowercase letters refer to column vectors. (.) $)^{H},(.)^{T}$ and $(.)^{+}$denote Hermitian transpose, transpose matrices and diagonal (with positive diagonal elements) matrix. respectively. $\mathbf{I}_{N}$ builds the $N \times N$ identity matrix. $(\mathbf{A})_{i j}$ represents the ( $i$-th, $j$-th) element of matrix $\mathbf{A}$ and $(\mathbf{A})_{K \times K}$ denotes a submatrix of $\mathbf{A}$ of size $K \times K$. The notation diag represents a diagonal matrix. If $\mathbf{B}$ is a $N \times N$ matrix, $\mathbf{A} \leq \mathbf{B}$ implies $\mathbf{A}-\mathbf{B} \leq 0$ is negative semidefinite. Finally, $\mathrm{E}\{$.$\} and \|$.$\| refer to the expected$ value operator and the Frobenius norm operators, respectively.

\section{SCENARIO DESCRIPTION}

\section{A. System Model}

Herein, the focus is on the forward link of a BMGP multibeam satellite system, where a single geosynchronous (GEO) satellite with multibeam coverage provides fixed broadband services to a large set of users with $N$ feeds and $K$ beams, configured corresponds to the MFB mode with $N>K$, in a frequency multiplexed fashion.

By employing a Time Division Multiplexing (TDM) scheme, at each time instant, a total of $K$ single antenna users, i.e. exactly one user per beam, is simultaneously served by a set of $F$ GWs. In this context, each GW serves a set of adjacent beams, and converts the corresponding FL signals into on-board feed signals through on-board BFN at the satellite. Let us assume that the GWs use a full frequency reuse pattern among the beams. Then, interbeam interference becomes the bottleneck of the whole system and employing precoding techniques is essential. Note that it is considered that the inter-FL interference can be neglected so that each GW is modelled as communicating with the satellite through an interferencefree and perfectly calibrated FL.

In this context, the received signal at the coverage area can be modeled as

$$
\mathbf{y}=\mathbf{H B x}+\mathbf{n},
$$

where $\mathbf{y}$ is a $K \times 1$ vector containing the symbols received by $K$ users, one per beam, at a given time instant. The $K \times 1$ vector $\mathbf{x}$ denotes the stacked transmitted signals at all the on-board feeds and the vector $\mathbf{n}$ of size $K \times 1$ contains the stacked zero mean unit variance Additive White Gaussian Noise (AWGN) such that $\mathrm{E}\left\{\mathbf{n n}^{H}\right\}=\mathbf{I}_{K}$. The BFN weights are included in matrix $\mathbf{B}$.

In the sequel, $\mathbf{H}$ is the overall $K \times N$ UL channel matrix whose element $(\mathbf{H})_{i j}$ represents the gain of the link between the $i$-th user (in the $i$-th beam) and the $j$-th satellite feed. The matrix $\mathbf{H}$ includes the propagation losses and radiation pattern, and as such is decomposed as [5]

$$
\mathbf{H}=\operatorname{diag}\left(\frac{1}{\sqrt{A}_{1}}, \ldots, \frac{1}{\sqrt{A}_{K}}\right) \mathbf{W},
$$

where $A_{k}$ denotes the propagation losses from the satellite to the $k$-th user. $\mathbf{W}$ is a $K \times N$ matrix which models the feed radiation patterns, the path loss and the received antenna gain. The $(k, n)$-th entry of $\mathbf{W}$ is modeled as

$$
(\mathbf{W})_{k n}=\frac{\sqrt{W_{R}} g_{k n}}{4 \pi \frac{d_{k}}{\lambda} \sqrt{k_{B} T_{R} B_{W}}}
$$

where $W_{R}$ denotes the user receive antennas power gain. $g_{k n}$ is referring to the gain (in power) from feed $n$ toward the $k$-th user, such that the respective feed transmit gain is $10 \log _{10}\left(\left|(\mathbf{W})_{k n}\right|^{2}\right)$ if expressed in $\mathrm{dBi}$. Finally, $d_{k}$ is the distance between the $k$-th user and the satellite, $\lambda$ the carrier wavelength, $k_{B}$ the Boltzmann constant, $T_{R}$ the receiver noise temperature, and $B_{W}$ the carrier bandwidth. The reader can refer to [5] for more details on (6).

To mitigate the inter-beam interference, we focus exclusively on the linear ZF precoding technique. This technique has been pointed out as an efficient method due to its complete interference rejection capabilities by the pseudoinverse of the channel, while preserving a low computational complexity [6]. To show the impact of precoding on the transmitted data, the vector $\mathbf{x}$ in (5) shall be decomposed as

$$
\mathbf{x}=\sqrt{\kappa} \mathbf{T s},
$$

where $\mathbf{s}$ is the transmit symbol vector and the $k$-th entry of $\mathbf{s}$ is the constellation symbol destined to the $k$-th user with $\mathrm{E}\left\{\mathbf{s s}^{H}\right\}=\mathbf{I}_{K}$. The matrix $\mathbf{T}$ denotes a $K \times K$ linear ZF precoding matrix. The scalar $\kappa$ is the power scaling factor and must adapt with

$$
\operatorname{trace}\left(\mathbf{B} \mathbf{T} \mathbf{T}^{H} \mathbf{B}^{H}\right) \leq P,
$$

where $P$ is the transmit power of $N$ feeds. Note that the transmit power constraint in (9) is set considering $\mathbf{B}$. Throughout this paper it is conceived that the power allocation mechanism is located at the array fed reflector system with $N$ embedded feeds.

The following section aims to provide the joint design of on-ground ZF precoding $\mathbf{T}$ and on-board BFN B in (5) for a BMGP architecture.

\section{B. BMGP configuaration}

As stated above, each GW serves a set of adjacent beams. It is referred to as a cluster of beams. A total number of $F \mathrm{GWs}$ and clusters are assumed, i.e. one $\mathrm{GW}$ per cluster. For instance, $m$-th ( $m=1, \ldots, F$ ) GW serves $K_{m}$ number of beams/users located in the $m$-th cluster such that $K=\sum_{m=1}^{F} K_{m}$. Without loss of generality, we consider an identical number of beams at each cluster, i.e.

$$
K_{1}=\ldots=K_{m}=\ldots=K_{F} .
$$

The objective of designing precoding in this context is to mitigate the intra-cluster and inter-cluster interference. The former arises from beams belonging to the same cluster while the latter relates to co-channel interference from beams served from other GWs. For mathematical convenience, we use $K_{-m}$ to denote the number of users located in other cluster different from $m$ with $K_{m}+K_{-m}=K$ and

$$
K_{-1}=\ldots=K_{-m}=\ldots=K_{-F} .
$$


The notation $N_{m}$ denotes the number of on-board feeds serving the $m$-th cluster, with $N=\sum_{m=1}^{F} N_{m}$. Again, without loss of generality, we let,

$$
N_{1}=\ldots=N_{m}=\ldots=N_{F} .
$$

The channel matrix $\mathbf{H}$ in (6) for a set of $F$ cluster/GWs can be decomposed as

$$
\mathbf{H}=\left(\mathbf{H}_{1}^{R}, \ldots, \mathbf{H}_{m}^{R}, \ldots, \mathbf{H}_{F}^{R}\right),
$$

with $\mathbf{H}_{m}^{R}$ of size $K \times N_{m}$ is the channel sub-matrix containing the contribution of the feeds assigned to the $m$-th GW. Then, we define

$$
\mathbf{H}_{m} \triangleq\left(\mathbf{H}_{m}^{R}\right)_{K_{m} \times N_{m}}
$$

as the $m$-th cluster submatrix obtained by selecting the corresponding $K_{m} \times N_{m}$ entries in the matrix $\mathbf{H}_{m}^{R}$. Besides,

$$
\mathbf{H}_{-m} \triangleq\left(\mathbf{H}_{m}^{R}\right)_{K_{-m} \times N_{m}}
$$

is the interference posed by the $N_{m}$ feeds on the adjacent clusters/beams of $m$. The $m$-th GW knows the CSI of both $\mathbf{H}_{m}$ and $\mathbf{H}_{-m}$.

In contrast to the previous works [7],[8], which have not contained a special structure on $\mathbf{B}$, a lower complexity payload is pursued here by operating with a block diagonal $\mathbf{B}^{1}$

$$
\mathbf{B}=\operatorname{diag}\left(\mathbf{B}_{1}, \ldots, \mathbf{B}_{m}, \ldots, \mathbf{B}_{F}\right),
$$

where the submatrices $\mathbf{B}_{m} \triangleq(\mathbf{B})_{N_{m} \times M_{m}},(m=1, \ldots, F)$ are of size $N_{m} \times M_{m}$. The term $M_{m}$ represents the number of signal streams to be transmitted in the FL from the $m$-th GW to the satellite, not necessarily equal to $N_{m}$, but possibly lower to save bandwidth, and such that $M_{m} \geq K_{m}$. For the sake of mathematical clarity, we assume $M_{m}=K_{m}$. The BFN transforms the $K_{m}$ FL signals into $N_{m}$ feed signals to serve the $m$-th cluster.

Precoding in (8) is also modeled by a block diagonal matrix, since each GW can process only its own streams. With this, the signal model in (5) for a set of $F$ GWs is written as

$$
\mathbf{y}=\mathbf{H B d i a g}\left(\sqrt{\kappa_{1}} \mathbf{T}_{1}, \ldots, \sqrt{\kappa_{m}} \mathbf{T}_{m}, \ldots, \sqrt{\kappa_{F}} \mathbf{T}_{F}\right) \mathbf{s}+\mathbf{n},
$$

where $\mathbf{y}=\left(\mathbf{y}_{1}, \ldots, \mathbf{y}_{m}, \ldots, \mathbf{y}_{F}\right)^{T}, \mathbf{s}=\left(\mathbf{s}_{1}, \ldots, \mathbf{s}_{m}, \ldots, \mathbf{s}_{F}\right)^{T}, \mathbf{B}$ is defined in (16) and $\mathbf{T}_{m}$ of size $K_{m} \times K_{m}$ refers to the precoder in the $m$-th GW. The scalar $\kappa_{m}$ is the power scaling factor in the $m$-th cluster, and has to comply with

$$
\kappa_{m}=\frac{P_{m}}{\operatorname{trace}\left(\mathbf{B}_{m} \mathbf{T}_{m} \mathbf{T}_{m}^{H} \mathbf{B}_{m}^{H}\right)},
$$

with $P_{m}$ denoting the total transmit power of $N_{m}$ feeds.

As an example, the signal model in (17) for three GWs and clusters can be expressed as follows

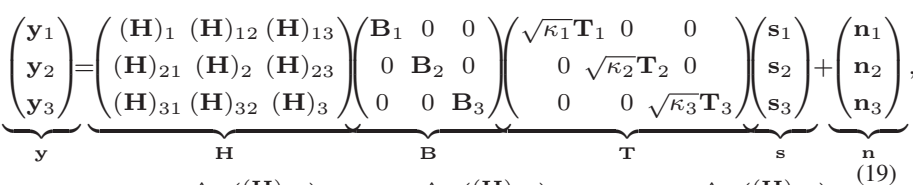

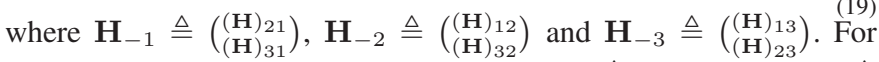
mathematical convenience, we also define $\mathbf{H}_{m} \triangleq(\mathbf{H})_{m}$; and $\mathbf{H}_{m i} \triangleq$ $(\mathbf{H})_{m i}$ which denotes the interference received by the $m$-th cluster from the $i$-th cluster, with $m \neq i$ and $i=1, \ldots,(F-1)$.

In this context, the signal model in (19) for the $m$-th cluster is written as

$$
\mathbf{y}_{m}=\sqrt{\kappa_{m}} \mathbf{H}_{m} \mathbf{B}_{m} \mathbf{T}_{m} \mathbf{s}_{m}+\sqrt{\kappa_{i}} \sum_{m \neq i}^{F-1} \mathbf{H}_{m i} \mathbf{B}_{i} \mathbf{T}_{i} \mathbf{s}_{i}+\mathbf{n}_{m}
$$

and the Signal to Noise plus Interference Ratio (SINR) of the $m$-th cluster is obtained by

$$
\operatorname{SINR}_{m}=\frac{\kappa_{m} \operatorname{trace}\left(\mathbf{H}_{m} \mathbf{B}_{m} \mathbf{T}_{m} \mathbf{T}_{m}^{H} \mathbf{B}_{m}^{H} \mathbf{H}_{m}^{H}\right)}{\kappa_{i} \sum_{i \neq m} \operatorname{trace}\left(\mathbf{H}_{m i} \mathbf{B}_{i} \mathbf{T}_{i} \mathbf{T}_{i}^{H} \mathbf{B}_{i}^{H} \mathbf{H}_{m i}^{H}\right)+1} .
$$

${ }^{1}$ Employing a block diagonal BFN in (16) instead of the proposed BFN in [8] establishes an affordable payload complexity through enforcing offblock diagonal elements of $\mathbf{B}$ to be zero (with no required processing in zero elements).
Then, the underlying problem for the $m$-th cluster can be formulated as

$$
\begin{array}{ll} 
& \max _{\mathbf{T}_{m}, \mathbf{B}_{m}} \operatorname{SINR}_{m} \\
\text { s.t. } & \operatorname{trace}\left(\mathbf{B}_{m} \mathbf{T}_{m} \mathbf{T}_{m}^{H} \mathbf{B}_{m}^{H}\right) \leq P_{m} .
\end{array}
$$

\section{PRECODING AND BFN IN BMGP}

\section{A. Precoding design}

In general, the design of $\mathbf{B}_{m}$ and $\mathbf{T}_{m}$ here aim to maximize $\mathrm{SINR}_{m}$ in (22) and guarantee:

Requirement.1. Nulling the inter-cluster interference through designing the precoding scheme $\mathbf{T}_{m}$ and obtains $\operatorname{trace}\left(\mathbf{H}_{m i} \mathbf{B}_{i} \mathbf{T}_{i} \mathbf{T}_{i}^{H} \mathbf{B}_{i}^{H} \mathbf{H}_{m i}^{H}\right)=\tau$, where

$$
\tau \triangleq \arg \min \left\{\operatorname{trace}\left(\mathbf{H}_{m i} \mathbf{B}_{i} \mathbf{T}_{i} \mathbf{T}_{i}^{H} \mathbf{B}_{i}^{H} \mathbf{H}_{m i}^{H}\right)\right\} .
$$

Requirement.2. Nulling the intra-cluster interference in addition to inter-cluster interference through the design of $\mathbf{T}_{m}$ and establishes $\operatorname{diag}\left\{\mathbf{H}_{m} \mathbf{B}_{m} \mathbf{T}_{m} \mathbf{T}_{m}^{H} \mathbf{B}_{m}^{H} \mathbf{H}_{m}^{H}\right\}$.

Requirement.3. Optimizing the power loading factor $\kappa_{m}$ in (18) and obtains $\arg \min \left\{\operatorname{trace}\left(\mathbf{B}_{m} \mathbf{T}_{m} \mathbf{T}_{m}^{H} \mathbf{B}_{m}^{H}\right)\right\}$, which is exploited through employing the joint design of $\mathbf{T}_{m}$ and $\mathbf{B}_{m}$. Note that only optimizing Requirement. 3 is performed at the payload aiming at preserving low computationally complex.

For a moment, let us consider the satellite payload works in the transparent mode, i.e. $\mathbf{B}=(\mathbf{I})_{N \times K}$, and the signal processing scheme is only done at the ground segment (i.e. at the GWs) such that the $\mathbf{T}_{m}$ precodes direcly a set of $K_{m}$ user signal into $N_{m}$ FL signals for the $m$-th cluster. In such a configuration, a promising block ZF precoding scheme to cope with inter- and intra-cluster interference is given by [9]

$$
\mathbf{T}_{m}=\mathbf{Q}_{m}^{H}\left(\mathbf{Q}_{m} \mathbf{Q}_{m}^{H}\right)^{-1}
$$

where

$$
\mathbf{Q}_{m} \triangleq \mathbf{H}_{m} \mathbf{V}_{-m}^{0} \mathbf{V}_{-m}^{0, H} \text {. }
$$

We write the Singular Value Decomposition (SVD) for the intercluster interfering channel as

$$
\mathbf{H}_{-m}=\mathbf{U}_{-m} \boldsymbol{\Sigma}_{-m} \mathbf{V}_{-m}^{H},
$$

with the matrix $\mathbf{V}_{-m}^{0}$ refers to the last $\left(N_{m}-K_{-m}\right)$ columns of matrix $\mathbf{V}_{-m}$ and denotes the null space of $\mathbf{H}_{-m}$. The power operator $\kappa_{m}$ in (18) for the proposed precoding scheme in (23) is obtained by

$$
\kappa_{p, m}=\frac{P_{m}}{\operatorname{trace}\left(\mathbf{T}_{m} \mathbf{T}_{m}^{H}\right)}=\frac{P_{m}}{\operatorname{trace}\left(\left(\mathbf{Q}_{m} \mathbf{Q}_{m}^{H}\right)^{-1}\right)} .
$$

Obviously, the proposed $\mathbf{T}_{m}$ in (23) fulfills the Requirement 1-3 and the $\mathrm{SINR}_{m}$ in (21) becomes

$$
\mathrm{SINR}_{m}=\kappa_{p, m} \text {. }
$$

Note that the sufficient condition for obtaining $\mathbf{V}_{-m}^{0}$ in (25) is to have $K_{-m}<N_{m}$.

Unfortunately, in case of $K_{-m}>N_{m}$, the null space matrix $\mathbf{V}_{-m}^{0}$ is empty. To cope with this circumstance, we propose the following design of $\mathbf{T}_{m}: \quad \mathbf{T}_{m}=\mathbf{Q}_{r, m}^{H}\left(\mathbf{Q}_{r, m} \mathbf{Q}_{r, m}^{H}\right)^{-1}$

with

$$
\mathbf{Q}_{r, m} \triangleq \mathbf{H}_{r, m} \mathbf{V}_{r,-m}^{0} \mathbf{V}_{r,-m}^{0, H} \mathbf{R}_{m}
$$

where $\mathbf{R}_{m}=\left(\begin{array}{c}(\mathbf{I})_{N_{m}} \times N_{m} \\ (\mathbf{I})_{K}-m \times N_{m}\end{array}\right)$.

For (28), the matrix $\mathbf{H}_{r, m}$ is the rescaled version of $\mathbf{H}_{m}$ and can be defined by $\mathbf{H}_{r, m}=\mathbf{H}_{m} \mathbf{R}_{m}^{H}$. Moreover, with the SVD of $\mathbf{H}_{-m} \mathbf{R}_{m}^{H}=\mathbf{U}_{r,-m} \boldsymbol{\Sigma}_{r,-m} \mathbf{V}_{r,-m}^{H}$, the matrix $\mathbf{V}_{r,-m}^{0}$ refers to the null space of $\left(\mathbf{H}_{-m} \mathbf{R}_{m}^{H}\right)$. The reader can refer to [5] and [10] for more methodologies to find the null space of a matrix in case of $K_{-m}>N_{m}$. It is worth to note here that the rest of the analytic analysis of this section is developed taking into account $K_{-m}<N_{m}$ degree of freedom at the payload. The sketch of the system analyzing with $K_{-m}>N_{m}$ is similar by adding a rescaling identity matrix $\mathbf{R}_{m}$.

In the presence of on-board BFN, the earlier results hold when $\mathbf{T}_{m}$ 
is replaced by $\mathbf{B}_{m} \mathbf{T}_{m}$. Further, we see that, it is possible to let $\mathbf{T}_{m}$ have the dimension of $K_{m} \times K_{m}$ and $\mathbf{B}_{m}$ to be $N_{m} \times K_{m}$. In that sense, we have,

$$
\mathbf{B}_{m} \mathbf{T}_{m} \rightarrow \mathbf{B}_{m} \mathbf{T}_{m}=\mathbf{Q}_{m}^{H}\left(\mathbf{Q}_{m} \mathbf{Q}_{m}^{H}\right)^{-1} \text {. }
$$

Employing $\left(\mathbf{B}_{m}^{H} \mathbf{B}_{m}\right)^{-1} \mathbf{B}_{m}^{H}$ at the both sides of (33) holds

$$
\mathbf{T}_{m}=\left(\mathbf{B}_{m}^{H} \mathbf{B}_{m}\right)^{-1} \mathbf{B}_{m}^{H} \mathbf{Q}_{m}^{H}\left(\mathbf{Q}_{m} \mathbf{Q}_{m}^{H}\right)^{-1} \text {. }
$$

To find an optimal design of BFN, we restrict the $\mathbf{B}_{m}$ in (31) to be

$$
\mathbf{B}_{m}^{H} \mathbf{B}_{m}=\mathbf{I}_{K_{m}},
$$

where this implies on-board BFN is constructed with orthonormal vectors. Given (32), the precoding in (30) is rewritten as

$$
\mathbf{T}_{m}=\mathbf{B}_{m}^{H} \mathbf{Q}_{m}^{H}\left(\mathbf{Q}_{m} \mathbf{Q}_{m}^{H}\right)^{-1} \text {. }
$$

The scalar $\kappa_{m}$ in (18) with the formulation of precoding in (33) as well as the assumption in (32) is recalculated as

$$
\kappa_{m}=\frac{P_{m}}{\operatorname{trace}\left(\mathbf{B}_{m}^{H} \mathbf{Q}_{m}^{H}\left(\mathbf{Q}_{m} \mathbf{Q}_{m}^{H}\right)^{-2} \mathbf{Q}_{m} \mathbf{B}_{m}\right)} .
$$

Let us consider the SVD of $\mathbf{Q}_{m}=\mathbf{U}_{m} \boldsymbol{\Sigma}_{m} \mathbf{V}_{m}^{H}$. Then, an optimal $\mathbf{B}_{m}$ can be constructed through the following matrices

$$
\mathbf{B}_{m}^{\star}=\mathbf{V}_{-m}^{0}\left(\begin{array}{c}
\mathbf{U}_{m} \\
\mathbf{z}
\end{array}\right) \quad m=1, \ldots, F
$$

where $\mathbf{z}$ of size $\left(\left(N_{m}-K_{-m}\right)-K_{m} \times K_{m}\right)$ is a zero matrix. With applying $\mathbf{B}_{m}^{\star}$ in (35), the Requirement.1-3 are satisfied and the $\operatorname{SINR}_{m}$ in (21) becomes

$$
\frac{\operatorname{SINR}_{m}=\kappa_{p, m}=}{\operatorname{trace}\left(\mathbf{B}_{m}^{\star, H} \mathbf{Q}_{m}^{H}\left(\mathbf{Q}_{m} \mathbf{Q}_{m}^{H}\right)^{-2} \mathbf{Q}_{m} \mathbf{B}_{m}^{\star}\right)}=\frac{P_{m}}{\operatorname{trace}\left(\left(\mathbf{Q}_{m} \mathbf{Q}_{m}^{H}\right)^{-1}\right)},
$$

where

$\operatorname{trace}\left(\mathbf{B}_{m}^{H} \mathbf{Q}_{m}^{H}\left(\mathbf{Q}_{m} \mathbf{Q}_{m}^{H}\right)^{-2} \mathbf{Q}_{m} \mathbf{B}_{m}\right)$

$\geq \operatorname{trace}\left(\mathbf{B}_{m}^{\star, H} \mathbf{Q}_{m}^{H}\left(\mathbf{Q}_{m} \mathbf{Q}_{m}^{H}\right)^{-2} \mathbf{Q}_{m} \mathbf{B}_{m}^{\star}\right)=\operatorname{trace}\left(\left(\mathbf{Q}_{m} \mathbf{Q}_{m}^{H}\right)^{-1}\right)$.

$$
\text { included in [11]. }
$$

However, while the UL channel varies, the optimal $\mathbf{B}_{m}$ in (35) should adapt with the UL channel variation, leading to a large complexity at the payload. Even though the UL channel appears to be variable, next section aims at finding a fixed design of $\mathbf{B}_{m}$ with respect to the UL channel perturbation.

\section{B. Fixed BFN design}

Towards generating a fixed $\mathrm{BFN}$, the constructing $\mathbf{B}_{m}$ includes the following steps:

(a) We begin by designing a fixed $\mathbf{B}_{m}$ which optimizes the power factor $\kappa_{m}$ in the calculated $\operatorname{SINR}_{m}$ (22) and fulfills the Requirement 3. To this end, for the $\kappa_{m}$ in (34), the objective problem can be formulated as

$$
\min _{\mathbf{B}_{m}} \operatorname{trace}\left(\mathbf{B}_{m}^{H} \mathbf{Q}_{m}^{H}\left(\mathbf{Q}_{m} \mathbf{Q}_{m}^{H}\right)^{-2} \mathbf{Q}_{m} \mathbf{B}_{m}\right)
$$

(b) We show that the obtained $\mathbf{B}_{m}$ in (a) fulfills Requirement 1-2. We consider the channel matrix $\mathbf{H}_{m}$ for the $m$-th $\mathrm{GW}$ can be decomposed as [12]

$$
\mathbf{H}_{m}=\hat{\mathbf{H}}_{m}+\Delta \mathbf{H}_{m}
$$

where $\hat{\mathbf{H}}_{m}$ represents the mean of channel response with respect to random user locations ${ }^{2}$ of $\mathbf{H}_{m}$. Perturbation matrix $\Delta \mathbf{H}_{m}$ models the difference between the actual value and its mean. Similarly, we formulate a perturbation model for the null subspace in (25):

$$
\mathbf{V}_{-m}^{0}=\hat{\mathbf{V}}_{-m}^{0}+\Delta \mathbf{V}_{-m}^{0} \text {. }
$$

It is conceived that the actual matrices $\mathbf{H}_{m}$ and $\mathbf{V}_{-m}^{0}$ respectively relay on the neighborhood of the nominal channel matrices $\hat{\mathbf{H}}_{m}$ and $\hat{\mathbf{V}}_{-m}^{0}$ that are known to the $m$-th GW. In particular, we consider

\footnotetext{
${ }^{2}$ Other random fluctuations are expected, even for fixed users, such as phase
} noise. that $\mathbf{H}_{m}$ and $\mathbf{V}_{-m}^{0}$ respectively belong to the uncertainty regions $\mathcal{H}_{m}=\left\{\left\|\mathbf{H}_{m}-\hat{\mathbf{H}}_{m}\right\| \leq \alpha \mathbf{\Delta H}_{m}\right\}$ and $\mathcal{V}_{m}=\left\{\left\|\mathbf{V}_{-m}^{0}-\hat{\mathbf{V}}_{-m}^{0}\right\| \leq\right.$ $\left.\alpha_{\Delta \mathbf{V}_{-m}^{0}}\right\}$, which are two spheres centered at $\alpha \Delta \mathbf{H}_{m}$ and $\alpha_{\Delta} \mathbf{V}_{-m}^{0}$. Interestingly, the channel model in (39) and (40) resembles the modeling of a MIMO system with imperfect CSI at the transmitter which has been solved as a worst case optimization problem in [13]. With this perspective, the worst case robust design is considered, which leads to a maxmin or minmax formulation. The corresponding minmax problem for the $m$-th cluster is intended to maximize the minimum of the trace $\left(\mathbf{B}_{m}^{H} \mathbf{Q}_{m}^{H}\left(\mathbf{Q}_{m} \mathbf{Q}_{m}^{H}\right)^{-2} \mathbf{Q}_{m} \mathbf{B}_{m}\right)$ in (38), i.e,

$$
\begin{gathered}
\min _{\mathbf{B}_{m}} \max _{\substack{\Delta \mathbf{H}_{m}, \mathbf{\Delta} \mathbf{V}_{-m}^{0} \\
\text { s.t. }}} \operatorname{trace}\left(\mathbf{B}_{m}^{H} \mathbf{Q}_{m}^{H}\left(\mathbf{Q}_{m} \mathbf{Q}_{m}^{H}\right)^{-2} \mathbf{Q}_{m}=\mathbf{I}_{K_{m}} .\right.
\end{gathered}
$$

Theorem 1. The derivation of the solution for (41) goes along the following logic:

(i) We set upper bounds $\alpha_{\Delta} \mathbf{H}_{m}$ and $\alpha_{\Delta} \mathbf{V}_{-m}^{0}$ for the spectral norms $\left\|\boldsymbol{\Delta} \mathbf{H}_{m}\right\|$ (i.e. $\left\|\boldsymbol{\Delta} \mathbf{H}_{m}\right\| \leq \alpha_{\Delta} \mathbf{H}_{m}$ ) and $\left\|\boldsymbol{\Delta} \mathbf{V}_{-m}^{0}\right\|$ (i.e. $\left\|\boldsymbol{\Delta} \mathbf{V}_{-m}^{0}\right\| \leq \alpha_{\Delta \mathbf{V}_{-m}^{0}}$ ), respectively.

(ii) An upper bound $\mathbf{Q}_{m}^{H}\left(\mathbf{Q}_{m} \mathbf{Q}_{m}^{H}\right)^{-2} \mathbf{Q}_{m} \leq \breve{\mathbf{S}}_{m}$ is found leading to trace $\left(\mathbf{B}_{m}^{H} \mathbf{Q}_{m}^{H}\left(\mathbf{Q}_{m} \mathbf{Q}_{m}^{H}\right)^{-2} \mathbf{Q}_{m} \mathbf{B}_{m}\right) \leq$ trace $\left(\mathbf{B}_{m}^{H} \breve{\mathbf{S}}_{m} \mathbf{B}_{m}\right)$. Note that $\breve{\mathbf{S}}_{m}$ is the upper bound of $\mathbf{Q}_{m}^{H}\left(\mathbf{Q}_{m} \mathbf{Q}_{m}^{H}\right)^{-2} \mathbf{Q}_{m}$ and is defined as

$\breve{\mathbf{S}}_{m} \triangleq\left(\left(\lambda_{\max }\left(\hat{\mathbf{H}}_{m} \hat{\mathbf{H}}_{m}^{H}\right)-\epsilon_{H}\right)^{+}\left(\lambda_{\max }\left(\hat{\mathbf{V}}_{-m}^{0} \hat{\mathbf{V}}_{-m}^{0, H}\right)-\epsilon_{V}\right)^{+}\right)^{-2}$,

where $\epsilon_{V} \triangleq\left(2 \alpha_{\Delta \mathbf{V}_{-m}^{0}} \sigma_{\max }\left(\hat{\mathbf{V}}_{-m}^{0}\right)+\alpha_{\Delta \mathbf{V}_{-m}^{0}}^{2}\right)$ and $\epsilon_{H} \triangleq$ $2 \alpha_{\Delta} \mathbf{H}_{m} \sigma_{\max }\left(\hat{\mathbf{H}}_{m}^{H}\right)+\alpha_{\Delta}^{2} \mathbf{H}_{m}$. The terms $\lambda_{\max }($.$) and \sigma_{\max }($.$) represent$ the maximum eigenvalue and singular value of the corresponding matrix, respectively.

(iii) With considering SVD of $\breve{\mathbf{S}}_{m}=\breve{\mathbf{L}}_{m} \breve{\mathbf{\Omega}}_{m} \breve{\mathbf{L}}_{m}^{H}$ and $\hat{\mathbf{H}}_{m} \hat{\mathbf{H}}_{m}^{H}=$ $\mathbf{U}_{H, m} \boldsymbol{\Sigma}_{H, m} \mathbf{U}_{H, m}^{H}$, it can be shown that

$$
\mathbf{B}_{m}=\breve{\mathbf{L}}_{m}\left(\begin{array}{c}
\mathbf{U}_{H, m} \\
\mathbf{w}
\end{array}\right)
$$

maximizes the minimum of the objective problem in (41). The matrix w of size $\left(N_{m}-K_{m}\right) \times K_{m}$ is a zero matrix.

Proof. The proof of Theorem 1 is omitted due to space limitation and is provided in [11].

Indeed, the proposed fixed $\mathbf{B}_{m}$ in (43) supports Requirement 1- 3 and, similar to the $\mathbf{B}_{m}$ (35), the $\mathrm{SINR}_{m}$ in (22) becomes $\mathrm{SINR}_{m}=$ $\kappa_{p, m}$.

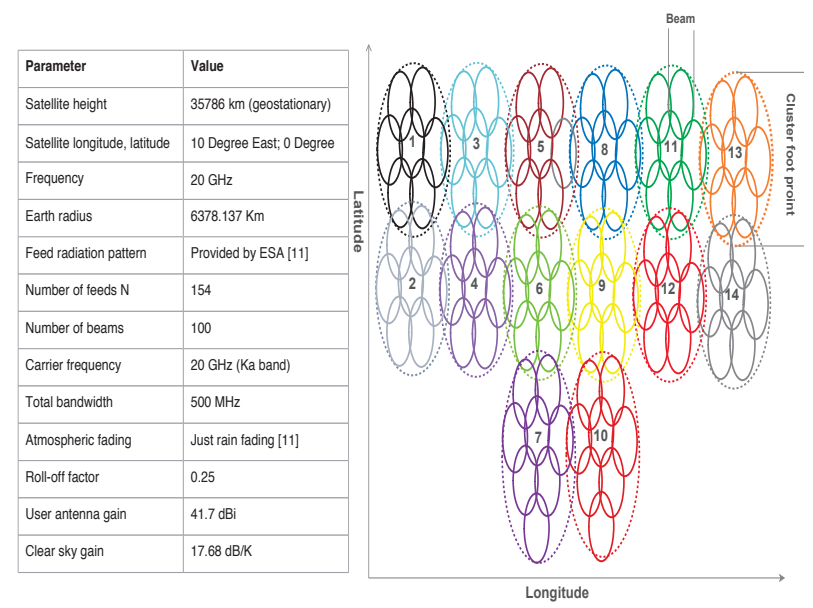

Fig. 1. UL simulation parameters (Left). A set of 14 clusters contour composed by 7 or 8 beams (Right). 


\section{NumERICAL RESUlTS}

To compare the performance of the proposed scenarios in this study, Monte Carlo simulations have been carried out. The simulation setup is based on an array fed reflector antenna/feed provided by European Space Agency (ESA) in the context of SatNexIII project with $N=154$ feeds and $K=100$ beams, at each time instant, which serve a single user per beam and spread over the whole Europe [12]. Results have been averaged for a total of 500 channel realizations. The detail of simulation parameters are collected in Fig. 1 (Left). Note that the channel fading statistics corresponds to the city of Rome.

As a performance metric, we compute the SINR for each user, after interference mitigation and then its throughput (bit/s) is inferred according to DVB-S2x standard for a packet error rate (PER) of $10^{-6}$ [14]. Note that this relationship has been obtained from [14] considering the PER curves. With this, the average total throughput at $m$-th cluster, $R_{\mathrm{DVB}-\mathrm{S} 2 \mathrm{X}, m}$, becomes

$$
R_{\text {DVB-S2X }, m}=\min _{q=1, \ldots, K_{m}} f_{\text {DVB-S2X }}\left(\mathbf{S I N R}_{m, q}\right)
$$

and the total average throughput can be calculated as

$$
R_{\text {DVB-S2X }}=\frac{2 B_{t o t}}{1+\rho} \sum_{m=1}^{F} R_{\text {DVB-S2X }, m},
$$

where $f_{\mathrm{DVB}-\mathrm{S} 2 \mathrm{X}}(\cdot)$ is function that provides the DVB-S2X spectral efficiency for a given SINR. The scalar $\rho$ is the Roll-off factor. By denoting $B_{\mathrm{UL}}$ as the total available bandwidth in the UL, $B_{\text {cluster }}=$ $B_{\mathrm{UL}} / F$ in (45) represents per cluster bandwidth. In this context, $B_{t o t}$ denotes the total available bandwidth for a set of $F$ GWs and can be calculated as

$$
B_{\text {tot }}=\frac{K}{N} F B_{\text {cluster }} .
$$

For a best practice and in order to clarify the performance of proposed BMGP architectures, we consider the following reference scenarios: 1) Upper-bound reference, a single GW multibeam architecture, i.e. $F=1$, is conceived where a linear $\mathrm{ZF}$ precoding is embedded at the GW aiming to mitigate inter-beam interference as well as the satellite works in transparent mode $\mathbf{B}=(\mathbf{I})_{N \times K}$. In this context, the mathematical expression of the ZF precoding scheme can be written as

$$
\mathbf{T}=\mathbf{H}^{H}\left(\mathbf{H H}^{H}\right)^{-1} .
$$

It is evident that the precoding in (47) can mitigate the interference in the UL. Even if the dimension employed in multiple GW architecture leads to drastically increase in the available FL bandwidth resource, however, this dimension limits the effectiveness of the interference mitigation techniques and therefore the system throughput is decreased with respect to the single GW scenario [5], [7].

2) Lower-bound reference, developing the BFN in BMGP network through the Discrete Fourier Transform (DFT) approach which is detailed in [7]. For the ground segment, the precoding in (28) is used.

\section{A. Results}

This section presents the simulation results related to the scenarios described in Section III. A total of $F=14 \mathrm{GWs}$ is considered so that each GW serves a cluster of 7 or 8 beams, i.e. $K_{m}=7$ or 8 . The contour of the beams and clusters distribution are depicted in Fig. 1 (Right). The number of employed feeds per GW is $N_{m}=11$. Note that the analysis in Section III assumes an identical number of beams per cluster; in practice, this can be easily extended to heterogeneous configurations as the one provided by ESA [12]. Remarkably, the effect of inter-FL interference is neglected.

The nature of the employed fed reflector antenna imposes the fact that $N_{m}<K_{-m}$ degree of freedom is available and the performance analysis of the proposed signal processing schemes in Sections III should be adapted with this constraint.
For the proposed BMGP architecture, the following schemes are compared:

(i) The upper bound reference.

(ii) ESA benchmark in BMGP (ESA-BMGP): the precoding in (28) and the BFN provided by ESA in the context of SatNex III project [12].

(iii) Adaptive Processing in BMGP (AP-BMGP): the precoding in (28) and the channel adaptive design of $\mathbf{B}$ in (35).

(iv) Fixed Processing in BMGP (FP-BMGP): the precoding in (28) and the non-channel adaptive design of $\mathbf{B}$ in (43).

(v) The lower bound reference.

Figure. 2 (Top-Left) depicts the evolution of the total average throughput (Gbps) as a function of $P$. As it is expected, the APBMGP provides a significant gain with respect to the upper bound reference scenario. This is due to its capability to adaptively cope with the inter- and intra-cluster interference at each GW. Since GWs employ the ZF precoding design in (28), the performance of proposed B characterized by Equation (43), i.e. FP-BMGP, is also illustrated in Figure 2 (Top-Left). This scheme shows performance improvement compared to ESA-BMGP and the lower bound; furthermore, it provides an acceptable results with respect to the AP-BMGP. This is due to the robustness of the proposed FP-BMGP while the UL channel varies by time.

Figure 2 (Top-Right) also compares the results of the proposed
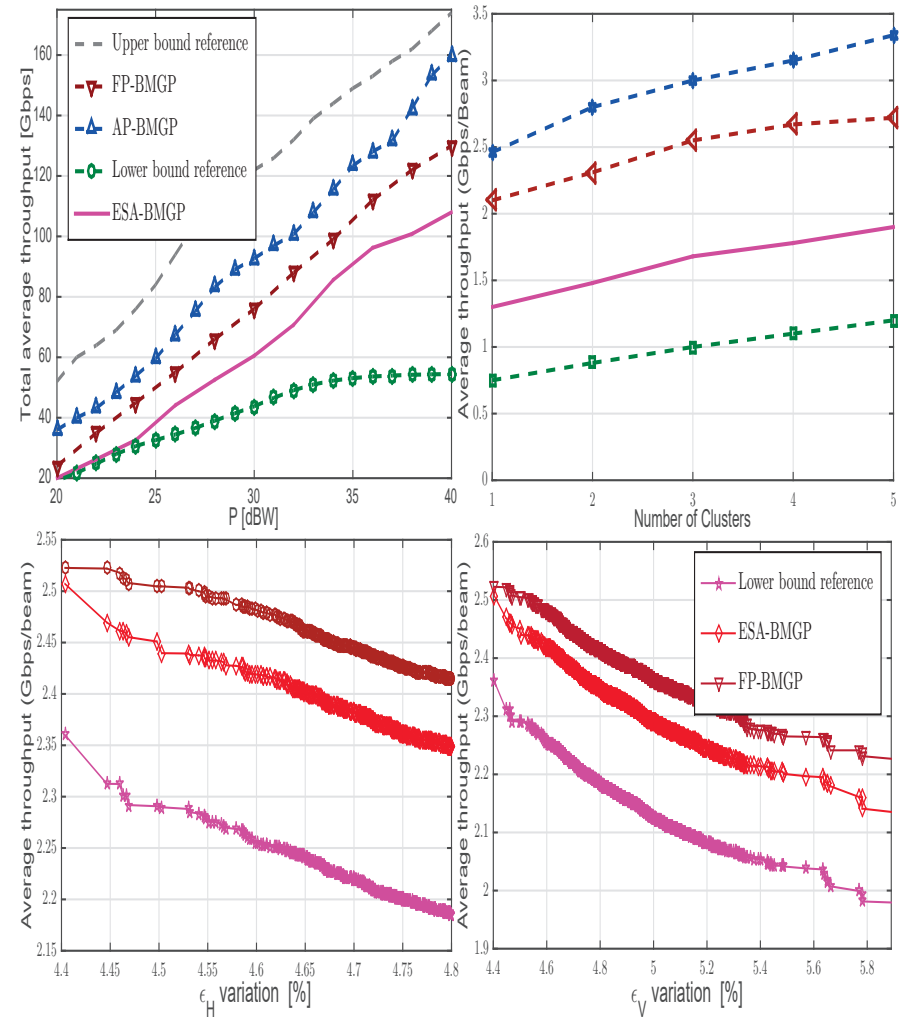

Fig. 2. (Top-Left) Average total throughput (Gbps) comparison for Scnearios (i)-(v) in section IV-A based on DVB-S2x MODCOD. (Top-Right) Average throughput per beam versus number of clusters. (Bottom-Left/Right) Throughput with respect to the UL channel variations $\epsilon_{H}$ and $\epsilon_{V}$.

schemes in (i)-(v) considering that each GW receives interference from 1 to 5 clusters. The transmit power is set to $P=35 \mathrm{dBW}$. Evidently, even with only one interfering cluster, the inter- and intra cluster interference increases. Nevertheless, the available bandwidth increases as the number of cluster increases (see eq.(45)-(46)). In this 
context, a performance improvement comes from the presented APand FP-BGMP with respect to the other scenarios.

The improvement can be also noticed from the corresponding robustness plots in Fig. 2 (Bottom-Left/Right). In this context, we study the impact of the UL channel variations on the FP-BGMP. Bearing in mind that the operators $\epsilon_{H}$ and $\epsilon_{V}$ in (42) determine the UL channel variation. It is worth to note that, the values of $\epsilon_{V}$ and $\epsilon_{H}$ are selected so that the feasibility of $\breve{\mathbf{S}}_{m}$ in Theorem 1 holds. It implies that

$$
\begin{gathered}
\left(\lambda_{\max }\left(\hat{\mathbf{H}}_{m} \hat{\mathbf{H}}_{m}^{H}\right)-\epsilon_{H}\right)^{+}>0, \\
\left(\lambda_{\max }\left(\hat{\mathbf{V}}_{-m}^{0} \hat{\mathbf{V}}_{-m}^{0, H}\right)-\epsilon_{V}\right)^{+}>0 .
\end{gathered}
$$

For a large value of $\epsilon_{H}$ or/and $\epsilon_{V}$ the expression (48) or/and (49) might become negative which changes the nature of the problem. In order to avoid this, the $\epsilon_{H}$ and $\epsilon_{V}$ have to be checked so that the right terms in (48) and (49) always remain positive. To overcome such excessive pessimism, it is necessary to include in $\epsilon_{H}$ and $\epsilon_{v}$ respectively the additional factor $\beta$ which should be found by numerical simulations. In such a condition, the expressions in (48) and (49) shall be rewritten by $\left(\lambda_{\max }\left(\hat{\mathbf{H}}_{m} \hat{\mathbf{H}}_{m}^{H}\right)-\beta \epsilon_{H}\right)^{+}>0$ and $\left(\lambda_{\max }\left(\hat{\mathbf{V}}_{-m}^{0} \hat{\mathbf{V}}_{-m}^{0, H}\right)-\beta \epsilon_{V}\right)^{+}>0$. The order of $\beta=10^{-2} \sim 10^{-3}$ which is obtained in 1000 channel realizations.

\section{CONCLUSION}

This paper dealt with the design of joint precoding and BFN in a multiple GW architecture. The analyzed multiple GW architecture suffers from i) a large intra-cluster, inter-cluster; and ii) high cost due to providing a fair balance between available FL resources and data demand growth.

Based on analytical formulations, the paper shows that implementing an appropriate on-board BFN (e.g. preferably non-channel adaptive) in a multiple $\mathrm{GW}$ architecture leads to optimize the required FL resources from $\frac{N}{F} \mathrm{~B}_{\text {beam }}$ in (3) to $\frac{K}{F} \mathrm{~B}_{\text {beam }}$ in (4) while imposing only a low computation complexity is targeted. Moreover, we developed a ZF precoding technique adapted with the proposed on-broad BFN in order to cope with the increased level of inter-cluster, intra-cluster such that each GW construct a part of the proposed full ZF precoding matrix.

\section{ACKNOWLEDGMENT}

This work was partially supported by the National Research Fund, Luxembourg under the project "PROSAT".

\section{REFERENCES}

[1] Daniel Minoli, "Innovations in satellite communications technology," in John Wiley \& Sons Inc. Hoboken, USA, 2015.

[2] L. Cottatellucci, M. Debbah, E. Casini, R. Rinaldo, R. Mueller, M. Neri and G. Gallinaro, "Interference mitigation techniques for broadband satellite system," in 24th AIAA International Satellite Communications Systems Conference, June 2006, USA.

[3] A. Gharanjik, B. Rao. Shankar, P. D. Arapoglou and B. Ottersten, "Gateway switching in $\mathrm{Q} / \mathrm{V}$ band satellite feeder links," in IEEE Letter of Communications, vol. 17, no. 7, pp. 1384-1387, July 2013.

[4] J. Tronc, P. Angeletti, N. Song, M. Haardt, and G. Gallinaro, "Overview and comparison of on-ground and on-board beamforming techniques in mobile satellite service applications," in International Journal of Satellite Communications and Networking, no. 4291-308, 2013.

[5] V. Joroughi, M. A. Vzquez and A. I. Prez-Neira, "Precoding in multigateway multibeam satellite systems," in IEEE Transactions on Wireless Communications, Vol. 15, No. 7, pp. 1-13, July 2016.
[6] SM Kay,'Fundamentals of statistical signal processing: estimation theory," in Prentice-Hall Inc. Upper Saddle River, 1993, USA.

[7] I. Thibault, B. Devillers, E. Candreva, F. Lombardo, A. VanelliCoralli, and G. Corazza, "Joint feeder-link bandwidth compaction and interference mitigation based on a hybrid space/ground processing architecture for a broadband multi-beam satellite," in International Journal of Satellite Communications and Networking, vol. 32, no. 2, pp. 107-125, 2014.

[8] V. Joroughi, M. A. Vazquez, A. Ana Perez-Neira and B. Devillers, "Design of an on board beam generation process for a multibeam broadband satellite system," in IEEE Transactions on Wireless Communications, Vol. 13, pp. 1-14, March 2017.

[9] B. Devillers and A. Perez-Neira, "Advanced interference mitigation techniques for the forward link of multi-beam broadband satellite systems," in Conference Record of the Forty Fifth Asilomar Conference on Signals, Systems and Computers, pp. 18101814, November 2011, USA.

[10] Y. Silva and A. Klein, "Linear transmit beamforming techniques for the multigroup multicast scenario," in IEEE Transactions on Vehicular Technology, vol. 58, no. 8, pp. 4353-4367, October 2009.

[11] V. Joroughi, S. Maleki, B. Shankar M.R., S. Chatzinotas, J. Grotz and B. Ottersten, Joint precoding and on-board beamforming for multiple gateway multibeam satellite systems, in Submitted to IEEE Transaction on Communication, December 2017. Available at: http://hdl.handle.net/10993/32264.

[12] Call of Order 2-Task 1, "Fair comparison and combination of advanced interference mitigation techniques," Satellite Network of Experts (SatNEx) 3, report of ESAcontract NO:23089/10/NL/CPL.

[13] D. P. Palomar, "A unified framework for communications through MIMO channels," in Ph.D. dissertation, Technical University of Catalonia (UPC), May 2003, Spain.

[14] ETSI EN 302 307-2: "Digital Video Broadcasting (DVB); Part 2: DVB-S2 Extensions (DVB-S2X)." 